\title{
A Simple Model of a Glow Discharge Electron Beam for Materials Processing
}

\author{
Javier Ignacio Etcheverry, Nélida Mingolo, Jorge J. Rocca, Senior Member, IEEE, \\ and Oscar Eduardo Martínez
}

\begin{abstract}
A simple semiempirical model of the electron beam generated by a pulsed cold cathode electron gun has been developed. The model describes analytically the observed self-focusing of the discharge and predicts the dynamical variation of the focal distance, in good agreement with experiments. This effect plays a major role in the determination of the effective duration of the energy pulse. The model was used to conduct simple calculations of energy thresholds for melting of solid materials, giving helpful insight on ranges of operation of this kind of electron gun for its application to material processing. A comparison with available experimental data for $\mathrm{Mg}_{70} \mathrm{Zn}_{30}$ samples is given.
\end{abstract}

\section{INTRODUCTION}

G LOW discharges can generate powerful electron beams without requiring heated cathodes. Several types of cold cathode glow discharges have been utilized to produce pulsed [1]-[4] as well as CW electron beams [5], [6]. The beams produced by these discharges have been used for various materials processing applications, including annealing [7]-[9], thin film deposition [10]-[12], and the etching of diamond films [13]. Recently, Mingolo et al. [14] applied a cold cathode electron gun to the amorphization of $\mathrm{Mg}-\mathrm{Zn}$ alloys. For the latter application we have utilized a particularly simple type of large cathode area pulsed discharge electron gun, that can produce high-power density focused electron beams following the secondary emission of electrons by ion bombardment of the cathode surface [3], [14].

In this type of electron gun the electrons are accelerated by the large electric field, typically several tens of $\mathrm{kV} / \mathrm{cm}$, that exists in the cathode sheath region of the glow discharge, adjacent to the cathode surface. At sufficiently high current density the self-generated magnetic field alters the electron trajectories, focusing the electron beam.

In this paper we report a semi-empirical model for the electron beam generated by this pulsed cold cathode electron gun, that allows us to describe the temporal and spatial characteristics of the electron beam. We show that the beam focuses at a distance from the cathode that depends on the

Manuscript received June 6, 1996; revised December 24, 1996. This work was supported in part by the Universidad de Buenos Aires under Grant IN063. Fundacion Antorchas and CONICET-Agentina, and under a grant from the Third World Academy of Sciences (TWAS).

J. I. Etcheverry and O. E. Martínez are with the Departamento de Matemática, Facultad de Ciencias Exactas y Naturales, Universidad de Buenos Aires, Pabellón I, Ciudad Universitaria, 1428 Buenos Aires, Argentina.

N. Mingolo is with the Departamento de Física, Facultad de Ingeniería, Universidad de Buenos Aires, Paseo Colón 850, 1063 Buenos Aires, Argentina.

J. J. Rocca is with the Electrical Engineering Department, Colorado State University, Fort Collins, CO 80523 USA.

Publisher Item Identifier S 0093-3813(97)04440-8.

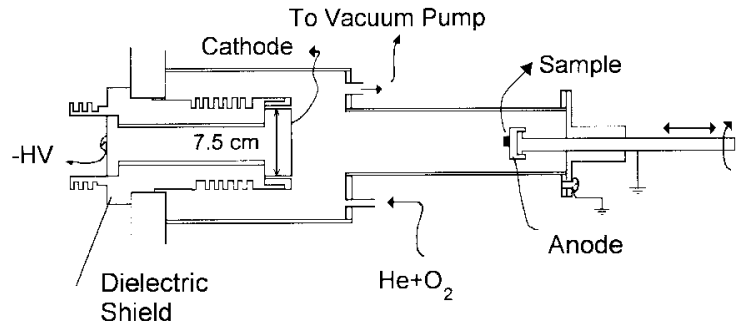

Fig. 1. Schematic diagram of the electron gun. The chamber is evacuated and filled with a mixture of $\mathrm{He}$ and $\mathrm{O}_{2}$. The sample is mounted on a translation and rotation shaft and the cathode $(7.5 \mathrm{~cm}$ in diameter) is made of aluminum with an oxide layer at the surface that is mantained due to the $\mathrm{O}_{2}$ in the gas atmosphere. For more details see Mingolo et al. [14].

glow discharge current and voltage. This self-focusing allows one to vary the dose of energy given to a material sample by simply adjusting the cathode-sample distance, at same discharge conditions.

Moreover, the model predicts an evolution of the focusing distance in time, as the current and voltage over the gun vary with time. This dynamic change of the focusing distance produces a rapid increase of the irradiated area in time, when the initially irradiated area is sufficiently small. It is shown that this effect allows one to obtain energy pulses much shorter than either the current or voltage pulses.

Simple formulas are deduced to describe the evolution of the energy flux delivered to the treated material, allowing to estimate melting or vaporization thresholds. These estimations are in good agreement with results from Mingolo et al. [14] for $\mathrm{Mg}_{70} \mathrm{Zn}_{30}$.

\section{Model of the Cold Cathode Electron Beam}

The cold cathode electron gun we are considering is similar to that developed by Ranea-Sandoval et al. [3] and used by Mingolo et al. [14] for the production of amorphous surfaces. This electron gun can produce high currents up to about $900 \mathrm{~A}$ at accelerating voltages of about $65 \mathrm{kV}$, in pulses of $0.2-30 \mu \mathrm{s}$.

It essentially consists on an aluminum cathode of $7.5 \mathrm{~cm}$ diameter enclosed in a dielectric shield (see Fig. 1). This cathode is introduced in a low pressure environment (usually an inert gas) and is connected to a high negative voltage source. The dischage circuit is shown in Fig. 2. With the sparkgap open, the high voltage source $V_{s}$ charges the capacitor $C$. When the spark-gap is triggered, the capacitor discharge produces a pulsed high-voltage glow discharge, with wellknown characteristics [3], [14]. 


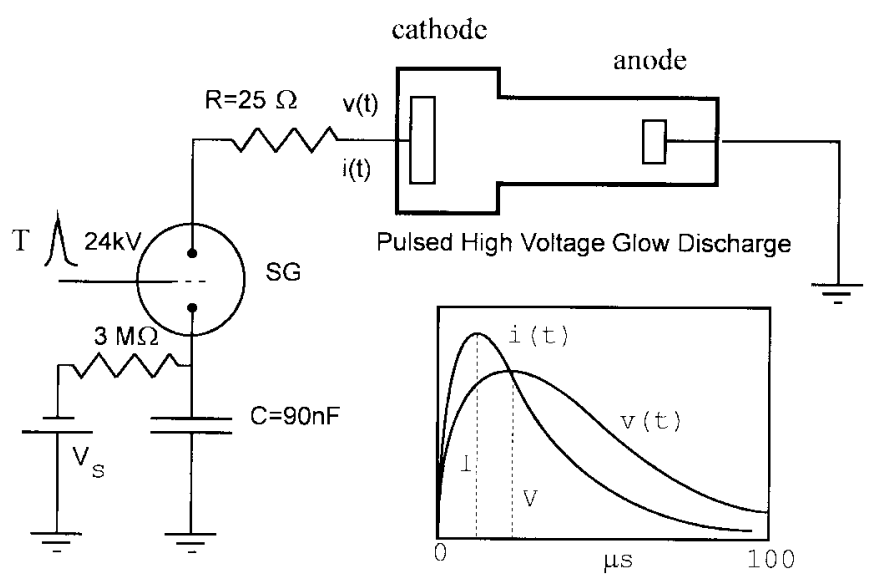

Fig. 2. Discharge circuit for the electron beam generation. The $90 \mathrm{nF}$ capacitor, initially charged at $V_{s}$, is discharged across the electron gun by triggering the spark-gap (SG). T: Trigger pulse. The cathode voltage and beam current are measured as described in [14].

A high electric space charge region, known as the cathode sheath, develops close to the cathode. This region is characterized by a positive charge and a strong electric field. Practically, all the discharge voltage drops in this region [15], producing electric fields of several tens of $\mathrm{kV} / \mathrm{cm}$ that accelerates ions toward the cathode and electrons in the opposite direction to form an electron beam. The electrons are emitted by the cathode when it is bombarded by the accelerated ions and fast neutral atoms. In order to obtain high current density pulses, it is necessary for the cathode to have a high secondary electron emission yield. Oxidized aluminum or magnesium cathodes are usually employed [3], [14]. A small fraction (5-10\%) of $\mathrm{O}_{2}$ is usually incorporated to the inert gas $(\mathrm{He})$ to maintain cathode oxidation.

The negative glow region, appearing farther from the cathode, occupies almost all the cathode-anode region (see [3]).

To conduct material processing studies the samples were placed in the axis of the electron beam path, and held in good electrical contact with ground, to minimize effects of space charge on the sample surface. The energy flux impinging on the sample surface can be modified by changing the initial voltage of the discharge, the current, or the irradiated surface. Changes in the discharge current are obtained by changing He pressure. The irradiated area can be modified by changing the anode-cathode distance, but depends also on the discharge voltage and gas pressure. Irradiation was performed in single shot mode, after five to ten stabilizing shots on a dummy target. In the next sections, a simple semiempirical model for the time and spatial behavior of the discharge will be presented.

\section{A. Simple Model of the Temporal Evolution of the Discharge Parameters}

Ranea-Sandoval et al. [3] showed that this type of electron gun can be operated on a wide range of voltages and currents, with upper limit set by arc formation. For an aluminum cathode in low pressure helium atmosphere also containing approximately 10 mtorr of $\mathrm{O}_{2}$, they showed that the peak current $I$ dependence on the initial discharge voltage $V$ and

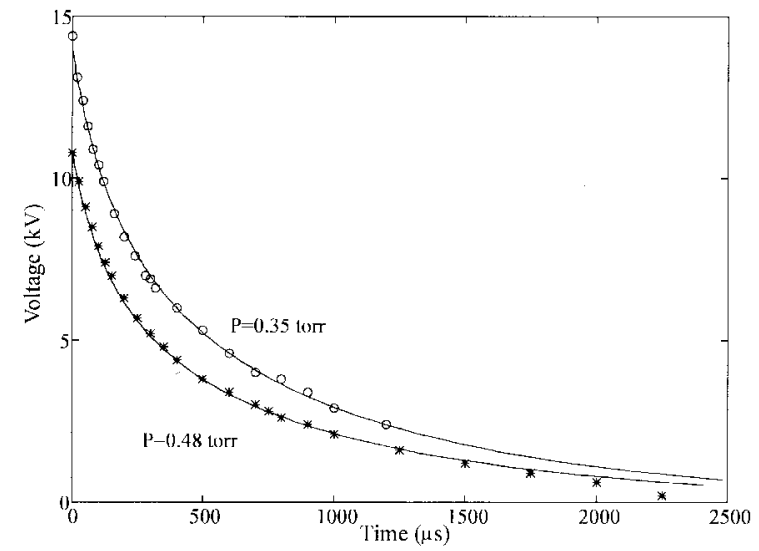

Fig. 3. Experimental (symbols) and predicted glow discharge voltage drop for $(0.350 \pm 0.01)$ torr and $(0.48 \pm 0.01)$ torr He pressure. $a=4.310^{-3}$ in units of $\mathrm{kV}$, A, torr.

helium pressure $p$ is well described by

$$
\begin{aligned}
& I=a V^{k} p^{m} \quad a=(4.8 \pm 0.1) 10^{-3} \\
& k=(3 \pm 0.15), \quad m=(2.2 \pm 0.2)
\end{aligned}
$$

where $I$ is in $\mathrm{A}, V$ in $\mathrm{kV}, p$ in torr, and $a$ is a constant that depends on the oxidation level of the cathode, on the repetition rate of the discharge, on the $\mathrm{O}_{2}$ partial pressure, etc. Herein, we show that this kind of simple relationships can also be used to describe the temporal evolution of the discharge parameters, in good agreement with experimental measurements. We will assume that the relation (1) between the current $i(t)$ and the voltage drop $v(t)$ holds for all times

$$
i(t)=a v(t)^{k} p^{m} \quad k=(3 \pm 0.15) \quad m=(2.2 \pm 0.2) .
$$

The constant $a$ that is determined experimentally is the only adjustable parameter in the analysis that follows.

The $i-v$ characteristics of the electron beam glow discharge (2) were used to solve the circuit shown in Fig. 2, neglecting the triggered spark-gap resistivity, and the current flowing through the $3 \mathrm{M} \Omega$ resistor. Integration of the resulting differential equation gives

$$
t=k C R \ln \left(\frac{v_{0}}{v}\right)+\frac{C}{a p^{m}(k-1)} \frac{1}{v_{0}^{k-1}}\left(\left(\frac{v_{0}}{v(t)}\right)^{k-1}-1\right)
$$

where $v_{0}$ is the initial voltage drop over the electron gun, and $R$ is the $25 \Omega$ resistor. The current can be then easily obtained from expression (2). Good agreement with experimental curves is obtained, as shown in Fig. 3, where the glow discharge voltage drop measured over a $13 \mathrm{k} \Omega$ resistor for two different pressures is compared with the voltage drop calculated following the previous discussion (and taking into account the $13 \mathrm{k} \Omega$ resistor). Note that the same value of $a\left(a=4.310^{-3}\right.$ in units of $\mathrm{kV}, \mathrm{A}$, torr) allows to fit both curves.

As a final remark, note that for small operating pressures ( $\sim 0.3$ torr), and typical values of $a(a \lesssim 0.01)$, the resistivity of resistor $R$ can be neglected

$$
k R \ll \frac{1}{a p^{m} v_{0}^{(k-1)}} .
$$


With this assumption the expressions that describe the evolution of the current and the voltage drop on the glow discharge are greatly simplified

$$
\begin{aligned}
& v(t)=v_{0}\left(1+\frac{a p^{m}(k-1) v_{0}^{k-1} t}{C}\right)^{-(1 / k-1)} \\
& i(t)=a p^{m} v_{0}^{k}\left(1+\frac{a p^{m}(k-1) v_{0}^{k-1} t}{C}\right)^{-(k / k-1)}
\end{aligned}
$$

The parameter $a$ can be easily calculated from (1), by measuring the initial glow discharge voltage and current, or fitting expression (5) to a given experimental $v(t)$ curve. An experimentally simpler approach, only requiring the measurement of the duration full-width-half maximum (FWHM) $\tau_{1 / 2}$ of the voltage pulse, can be obtained from expression (5)

$$
a=0.13 \frac{C}{p^{m} v_{0}^{k-1} \tau_{1 / 2}}
$$

where $C$ is in $F, \tau_{1 / 2}$ in seconds, $v_{0}$ in $\mathrm{kV}$, and $p$ in torr. The above expressions are used in Section III to compute the time dependence of the electron beam focusing distance, energy density deposition, and melting threshold.

\section{A. A Simple Model of the Spatial Shape of the Discharge}

In the negative glow region, the electrical field is essentially zero, there is no net charge, and the current is due to the flux of energetic beam electrons. Considering this current is in direction $-\hat{z}$, and assuming there exists rotational symmetry on $z$-axis, the generated magnetic field is in $-\hat{\phi}$ direction. The radially inwards force exerted by the magnetic field over an electron with velocity $\boldsymbol{u}=u \hat{z}$ is $\boldsymbol{F}=q \boldsymbol{u} \times \boldsymbol{B}$. In this way the electron beam self-focuses by Lorentz force, in a way given by the total current and the velocity of the electrons.

A simple model for this phenomenon can be developed, that agrees adequately with existing experimental data. Suppose that the electron beam occupies the region

$$
0 \leq r \leq r(z) \quad 0 \leq z \leq d
$$

where the cathode is assumed to be at $z=0, r(z)$ is the radius of the beam at distance $z$ from the cathode and $d$ is the anodecathode distance. Denote with $i$ the total current and with $E$ the electron energy. Let $m$ and $e$ be the electron mass and charge, respectively, and $\mu_{0}=4 \pi \mathcal{K}=1.2510^{-6} \mathrm{~N} / A^{2}$ the vacuum magnetic permeability.

The electron velocity is given by $\boldsymbol{u}=(d r / d t, 0, d z / d t)$. The nonrelativistic Newton equations for an electron initially on the limit of the electron beam can be written

$$
\begin{aligned}
& m \frac{d^{2} r}{d t^{2}}=-\frac{\mu_{0} i e}{2 \pi m r} \frac{d z}{d t} \\
& m \frac{d^{2} z}{d t^{2}}=\frac{\mu_{0} i e}{2 \pi m r} \frac{d r}{d t}
\end{aligned}
$$

where the magnetic field $B=\left(\mu_{0} i\right) /(2 \pi r)$ was calculated using the Ampère theorem, the initial conditions are

$$
r(0)=R_{0} \quad \dot{r}(0)=0 \quad z(0)=0 \quad \dot{z}(0)=u_{0}
$$

where $R_{0}$ is the cathode radius, and $u_{0}$ is the initial velocity (assumed to be normal to the cathode surface). The initial value of the electron velocity (velocity of the electrons at the cathode sheath-negative glow boundary) can be written nonrelativistically as

$$
u_{0}=c \sqrt{2 E / m c^{2}}=\sqrt{\frac{2 e v}{m}}
$$

where the energy $E$ is measured in $\mathrm{MeV}, m c^{2}=0.511 \mathrm{MeV}, c$ is the light velocity, and $v$ is the accelerating voltage. The main assumptions here are that the whole voltage drop occurs in the cathode sheath, a good approximation for this type of high voltage glow discharges, and that the accelerated electrons obtain all this energy $(E=e v)$. The effects of collisions of the beam electrons with the gas atoms are neglected. The latter approximation is justified by the fact that at the voltage and pressure of interest, the collision mean-free paths are long and the energy lost per collision is small compared with the initial energy of the beam electrons.

An explicit form for the trajectory $r(z)$ is obtained by eliminating time between the expressions for $r(t)$ and $z(t)$ given by the solutions of the Newton equations (9), (10). The focusing distance is then obtained from the curve $r(z)$.

Direct integration of (9) and (10) is difficult and leads to too complex expressions. Instead, Newton equations have been solved numerically, to obtain accurate results on the spatial characteristics of the electron beam. Alternatively, by expanding $r(t), z(t)$ in Taylor series and replacing in (9), (10), and (11), the following expressions are obtained:

$$
\begin{aligned}
& r(t) \sim R_{0}-\frac{1}{2} \frac{\mu_{0} i e}{2 \pi m} \frac{u_{0}}{R_{0}} t^{2} \\
& z(t) \sim u_{0} t-\left(\frac{\mu_{0} i e}{2 \pi m}\right)^{2} \frac{u_{0}}{R_{0}^{2}} t^{3} .
\end{aligned}
$$

Eliminating $t$ between these two equations gives

$$
\begin{aligned}
r(z) & \sim R_{0}\left(1-\frac{\mu_{0}}{4 \pi} \frac{i}{\sqrt{v}} \sqrt{\frac{e}{2 m}} \frac{z^{2}}{R_{0}^{2}}\right) \\
& =R_{0}\left(1-\frac{z^{2}}{\left(z^{*}\right)^{2}}\right)
\end{aligned}
$$

where $z^{*}$ is the cathode-focus distance

$$
z^{*} \sim R_{0} \sqrt{\frac{4 \pi m u_{0}}{\mu_{0} i e}}=R_{0} \frac{v^{1 / 4}}{i^{1 / 2}}\left(\frac{2 m}{e}\right)^{1 / 4} \sqrt{\frac{4 \pi}{\mu_{0}}} .
$$

The value of $z^{*}$ given by this analytical expression is slightly greater than the value obtained from the numerical computation. Fig. 4 shows the predicted variation of the electron beam radius for a typical discharge.

Table I illustrates the good agreement between the experimental focalization distance results from [3], and the corresponding values obtained with the present discharge model. $z_{\text {anal }}^{*}$ stands for the result obtained from (16), while $z_{\text {num }}^{*}$ was obtained by numerically solving the Newton equations (9) and (10). Cathode radius: $3.75 \mathrm{~cm}$.

Formula (16) shows an interesting competition phenomenon: as the discharge current diminishes, so it does the magnetic field. As a result the force tending to collapse the 


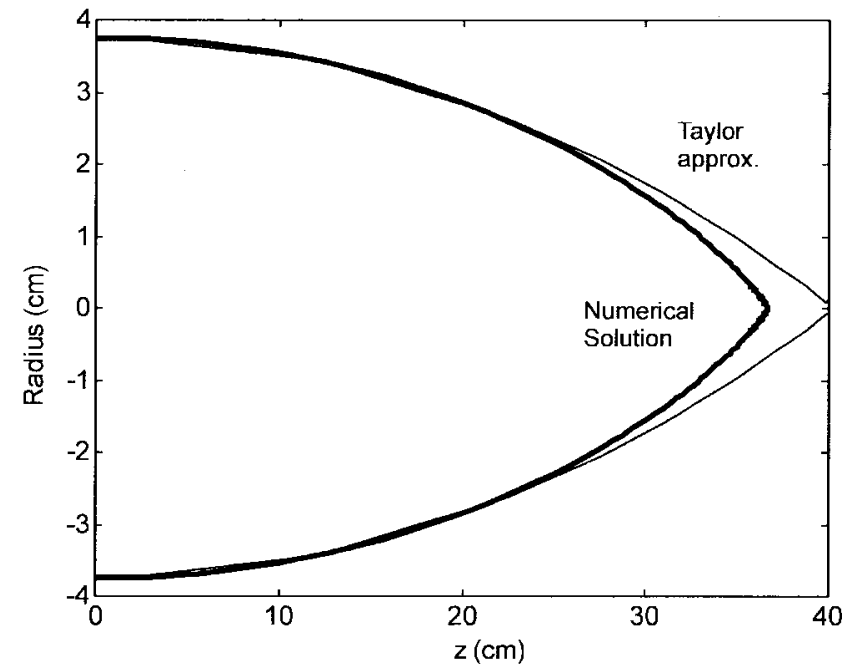

Fig. 4. Calculated variation of the electron beam radius for a typical discharge. The numerical solution (thick line) and the results of the Taylor approximation (thin line) are shown. Voltage drop: $22.5 \mathrm{kV}$, current: $41 \mathrm{~A}$, $R_{0}=3.75 \mathrm{~cm}$.

TABLE I

Electron Beam Focusing Distance. Experimental DATA FROM [3]. ANALYTICAL RESUltS FROM EXPRESSION (16). Numerical Results ObTained by Solving Numerically Newton Equations (9) AND (10) With Initial Data (11). RADIUS OF THE CATHODE: $3.75 \mathrm{~cm}$

\begin{tabular}{ccccc}
\hline Voltage & Current & $z_{\mathrm{cxp}}^{*}$ & $z_{\text {anal }}^{*}$ & $z_{\text {num }}^{*}$ \\
$72 \mathrm{kV}$ & $250 \mathrm{~A}$ & $20 \mathrm{~cm}$ & $22.7 \mathrm{~cm}$ & $20.8 \mathrm{~cm}$ \\
$72 \mathrm{kV}$ & $300 \mathrm{~A}$ & $17.5 \mathrm{~cm}$ & $19.5 \mathrm{~cm}$ & $17.8 \mathrm{~cm}$ \\
\hline
\end{tabular}

electron beam is smaller and the focus should move away from the cathode surface. On the other hand, as the current diminishes, also the accelerating voltage and the initial electron velocity are smaller, and consequently the focus should move toward the cathode. From expressions (2) and (16), it is clear that

$$
z^{*} \sim v^{-5 / 4} \text {. }
$$

In this way, we expect that the focus should move away as the discharge evolves in time. This dynamical evolution of the focalization distance is of significant interest, because if the initially irradiated spot on the target is small enough, the increase in the irradiated area as the beam defocuses is the major determinant of the reduction in the energy flux pulse width. An approximate analysis of these situations is given in the next section.

\section{Shortening of The ENERgy Pulse}

Under the assumptions of the preceding section, the total beam energy impinging on the surface of a sample in the unit time can be calculated as $i(t) v(t)$. Taking into account the percentage of energy carried away by backscattered electrons (reflectivity $\mathcal{R}$ ), the total energy incoming to the sample is $(1-\mathcal{R}) i(t) v(t)$. The energy flux deposited into the material is

$$
\Phi(t)=(1-\mathcal{R}) i(t) v(t) \frac{1}{S(t)}
$$

where $S(t)$ is the area being irradiated at time $t$, that can be calculated from (15)

$$
S(t)=\pi R_{0}^{2}\left(1-\frac{\mu_{0}}{4 \pi} \frac{i(t)}{\sqrt{v(t)}} \sqrt{\frac{e}{2 m}} \frac{d^{2}}{R_{0}^{2}}\right)^{2}
$$

where $d$ is the cathode-sample distance.

Based on experimental observations we assume that the energy flux inside the irradiated area is uniform for currents less than 200 A. At higher currents, experiments show the onset of a mini-beam with an extremely high current density and very small radius. This regime has been well characterized by Ranea-Sandoval et al. [3].

Let $v_{0}, i_{0}$ be the initial voltage drop and current, $r_{0}$ the initial radius of the irradiated spot, and $R_{0}$ the cathode radius. For a typical application to material processing, involving a high-energy dose, we have

$$
\gamma=\frac{r_{0}}{R_{0}} \ll 1
$$

Mingolo et al. for instance, report amorphization of $\mathrm{Mg}_{70} \mathrm{Zn}_{30}$ for an irradiated spot of about $0.36 \mathrm{~cm}$ radius, the radius of the cathode being $3.75 \mathrm{~cm}$ (this corresponds to $\gamma \sim 0.1$ ). It is shown below that for this situation the characteristic time for energy flux decay is much smaller than a characteristic time for current or voltage decay and that it is given by a characteristic time for irradiated area growth.

From (5) and (6), it is clear that a characteristic time for the discharge of the capacitor $C$ through the glow discharge is

$$
\tau_{D}=\frac{C}{a p^{m}(k-1) v_{0}^{k-1}} \text {. }
$$

To simplify the analysis of the expression for $\Phi(t)$, it is worth to adimensionalize the expressions for $v(t), i(t), S(t)$. Dimensionless variables are designated with a tilde. Voltage drop and current are adimensionalized with $v_{0}, i_{0}$. Reescaling times with $\tau_{D}$, expressions (5) and (6) are written in the simple form

$$
\begin{aligned}
& \tilde{v}(t)=(1+\tilde{t})^{-1 /(k-1)} \\
& \tilde{i}(t)=(1+\tilde{t})^{-k /(k-1)} .
\end{aligned}
$$

From expression (16), we get

$$
z^{*}(\tilde{t})=R_{0} \frac{v(t)^{1 / 4}}{i(t)^{1 / 2}}\left(\frac{2 m}{e}\right)^{1 / 4} \sqrt{\frac{4 \pi}{\mu_{0}}}=z_{0}^{*} \frac{\tilde{v}(\widetilde{t})^{1 / 4}}{\tilde{i}(\tilde{t})^{1 / 2}}
$$

where $z_{0}^{*}$ is the initial distance from the cathode to the focus. Reescaling lengths with $z_{0}^{*}$, we have

$$
\tilde{z}^{*}(\tilde{t})=\frac{\tilde{v}(\tilde{t})^{1 / 4}}{\tilde{i}(\tilde{t})^{1 / 2}}=(1+\tilde{t})^{(1 / 2)(1 / k-1)(k-(1 / 2))} \sim 1+\frac{5}{8} \tilde{t} .
$$

The reescaled radius at the sample surface can be calculated according to (15)

$$
\tilde{r}(\tilde{t})=\tilde{R}_{0}\left(1-\frac{\tilde{d}^{2}}{\left(\tilde{z}^{*}\right)^{2}}\right) \sim \tilde{R}_{0}\left(1-\tilde{d}^{2}\left(1-\frac{5}{4} \tilde{t}\right)\right) .
$$


Using that $\tilde{r}(0)=\tilde{r}_{0}$, we get

$$
1-\tilde{d}^{2}=\frac{\tilde{r}_{0}}{\tilde{R}_{0}}=\gamma \Leftrightarrow \tilde{d}^{2}=(1-\gamma)
$$

Rewriting (26)

$$
\tilde{r}(\tilde{t})=\tilde{r}_{0}+\tilde{R}_{0}(1-\gamma) \frac{5}{4} \tilde{t} \sim \tilde{r}_{0}+\tilde{R}_{0} \frac{5}{4} \tilde{t} .
$$

In this way, we see that the radius of the irradiated spot increases linearly in time, with a duplication time of

$$
\tau_{R}=\frac{4}{5} \gamma \tau_{D} \ll \tau_{D} \quad \text { if } \quad \gamma \ll 1
$$

The energy flux can now be expressed for $t \ll \tau_{D}$ as

$$
\Phi(t) \sim(1-\mathcal{R}) i_{0} v_{0} \frac{1}{\pi\left(r_{0}+R_{0} \frac{5}{4} \frac{t}{\tau_{D}}\right)^{2}} .
$$

The width FWHM of the energy flux pulse is then given by a characteristic area duplication time

$$
\tau_{E}=(\sqrt{2}-1) \tau_{R} \sim 0.33 \gamma \tau_{D}
$$

This shows that if the initial irradiated area is small enough, the obtained energy flux pulse is much shorter than both the current and voltage pulses.

For the cited Mingolo et al. [14] data an amorphous sample was obtained for a pulse with $v_{0}=22.5 \mathrm{kV}, i_{0}=41 \mathrm{~A}$, $\tau=23 \mu$ sWHM, $S=0.4 \mathrm{~cm}^{2}$. For these parameters, and a $\gamma=0.097$, the model gives a duration of the energy pulse of $1 \mu \mathrm{s}$ FWHM, much shorter than what was expected from the current pulse duration.

\section{Estimates of Thresholds for Melting}

The thermal problem associated with the deposition of the energy of this type of electron beam in the material is essentially one-dimensional (1-D) because the thermal diffusion length for a typical $20 \mu$ s pulse is much smaller than typical dimensions of the irradiated spot.

In the following it will be assumed that the penetration depth of the incident electrons is also smaller than a typical thermal diffusion length. In this situation, the melting threshold can be estimated without a detailed picture of the energy deposition process. This assumption is valid for heavy elements in the whole range of energies (10-100 keV) and pulse width durations (1-20 $\mu \mathrm{s})$ of interest for this type of electron beams, but for light elements is only valid at relatively long pulses and small energies. In aluminum, for instance, the penetration depth of $20 \mathrm{keV}$ electrons is about $1 \mu \mathrm{m}$ and that of $100 \mathrm{keV}$ electrons is about $40 \mu \mathrm{m}$ [16]. For $1 \mu \mathrm{s}$ pulses, the typical diffusion length is approximately $13 \mu \mathrm{m}$.

For a material irradiated with a constant energy flux of duration $\tau$, the threshold energy flux for melting can be written [17]

$$
\Phi_{T}=\frac{\sqrt{\pi}}{2}\left(T_{f}-T_{0}\right) \frac{\sqrt{\kappa \rho c_{p}}}{\sqrt{\tau}}
$$

where $T_{f}$ and $T_{0}$ are the fusion and initial temperatures respectively; $\kappa$ is the thermal conductivity, $\rho$ the density, and $c_{p}$ the specific heat.
In what follows the voltage thresholds for surface melting are obtained as a function of the operating parameters $a, p, d$, for $\gamma \ll 1$, and taking into account the predicted shortening of the energy pulse.

Approximating the energy flux for $\gamma \ll 1$ by a "square" pulse of intensity $i_{0} v_{0}(1-\mathcal{R}) / \pi / r_{0}^{2}$ and width $\tau_{E}$, and requiring that the approximate intensity be greater than the threshold intensity given by (32), we arrive to the relationship for the values of $\gamma$ and voltage at the melting threshold (denoted by $\gamma_{T}, v_{T}$, respectively)

$$
v_{T}=\left(6.8\left(T_{f}-T_{0}\right) \sqrt{\kappa \rho c_{p}} \frac{R_{0}^{2}}{(1-\mathcal{R})} \frac{1}{\sqrt{C a p^{m}}}\right)^{1 / 3} \sqrt{\gamma_{T}} .
$$

The physical meaning of the threshold value $\gamma_{T}$ is simple: the initial radius $r_{0}$ must be smaller than $\gamma_{T} R_{0}$ in order to achieve surface melting. Note that $\gamma_{T}$ depends itself on $v_{T}$, so further manipulations of expression (33) are needed in order to isolate $v_{T}$.

The factor multiplying $\sqrt{\gamma_{T}}$ in the right side can be recognized as the initial voltage needed to achieve melting over an area equal to the cathode area (i.e., if there were neither focusing of the electron beam, nor reduction of the pulse duration produced by the dynamical focusing). In this way, we will denote this factor with $v_{T}^{0}$.

The calculation below can be made simpler and more meaningful by introducing, for a given sample-cathode distance $d$, coefficient $a$ and pressure $p$, the voltage drop $v_{C}$ necessary in order to have the electron beam focused at $d$

$$
v_{C}=\left(\frac{R_{0}}{d} \frac{1}{\sqrt{a p^{m}}}\left(\frac{2 m}{e}\right)^{1 / 4} \sqrt{\frac{4 \pi}{\mu_{0}}}\right)^{4 / 5} .
$$

Now, we can write down the relationship between $\gamma_{T}$ and $v_{T}$. Rewriting expression (15) by putting the current $i$ as a function of the voltage $v$, we get

$$
r_{0}=R_{0}\left(1-\left(\frac{v_{0}}{v_{C}}\right)^{5 / 2}\right) .
$$

For small $\gamma$, we have

$$
\frac{v_{0}}{v_{C}} \sim 1-\frac{2}{5} \gamma
$$

Rewriting (33) for small $\gamma_{T}$, we can replace $v_{T}$ by $v_{C}$, arriving to the following expression for the threshold $\gamma_{T}$ value:

$$
\gamma_{T}=\left(\frac{v_{C}}{v_{T}^{0}}\right)^{2} .
$$

Written in terms of voltage thresholds, by using (36), we finally get

$$
v_{T}=v_{C}\left(1-\frac{2}{5}\left(\frac{v_{C}}{v_{T}^{0}}\right)^{2}\right) .
$$

Melting can then be achieved for a voltage range, of relative width given by $\frac{2}{5}\left(v_{C} / v_{T}^{0}\right)^{2}$ and absolute value given by $v_{C}$. Expression (38), combined with definitions of $v_{C}, v_{T}^{0}$, gives also detailed information on the variation of the voltage range 
for surface melting with parameters $d$ and $a p^{m}$. It can be easily seen that for small cathode-sample distances $d$, or for small values of $a p^{m}$ the relative width of the available voltage range for surface melting of the irradiated sample is greater.

We have applied formula (38) to the results of Mingolo et al. for $\mathrm{Mg}_{70} \mathrm{Zn}_{30}$ amorphization. In these experiments amorphization is achieved for a $21 \mathrm{~J}$ pulse $(22.5 \mathrm{kV}$ initial voltage drop, and 41 A peak current). Pulses of 10,12 , and 15 $\mathrm{J}$ (corresponding to initial voltages of 18,18 , and $20 \mathrm{kV}$, respectively) did not produce amorphization.

According to the presented model, we get from (2) $a p^{m}=$ $3.610^{-3}$ in units $\mathrm{kV}$, A. From the radius of the irradiated area $(0.36 \mathrm{~cm})$ and the radius of the cathode used in these experiments, a value of $\gamma=0.09$ is obtained. For the above parameters the expression (36) gives $v_{C}=23.4 \mathrm{kV}$.

Thermal parameters for $\mathrm{Mg}_{70} \mathrm{Zn}_{30}$ are (see [14])

$$
\kappa=1 \frac{J}{\mathrm{~cm} \mathrm{sK}} \quad \rho=2.94 \frac{g}{\mathrm{~cm}^{3}} \quad c_{p}=0.67 \frac{J}{\mathrm{gK}} \quad T_{f}=613 \mathrm{~K} .
$$

The reflectivity is estimated in 0.14 for $20 \mathrm{keV}$ electrons [16] and does not depend too much on electron energy.

For these values, $v_{T}^{0}=44.1 \mathrm{kV}$. Accordingly, we get

$$
v_{T}=20.7 \mathrm{kV}
$$

in close agreement with Mingolo et al. results.

\section{CONCLUSIONS}

A simple semi-empirical model of the evolution of the spatio-temporal characteristics of the electron beam produced by a cold cathode high-voltage glow discharge is described. Simple analytical expressions are given that can be used to estimate the electron beam characteristics that are important for high-energy density material processing, including irradiated spot size, focal distance, energy density, and threshold energy density for melting. The only adjustable parameter used in this model can be determined with the simple measurement of the voltage drop for a single shot (after a few stabilizing shots).

Comparison with helium glow discharge experiments show that the model accurately describes the time evolution of the discharge voltage and current, the self focusing of the electron beam, and the threshold energy for melting.

Expressions (38), (33), and (34) of the threshold voltage necessary for melting show that the sensitivity to voltage variations is reduced at high discharge voltages and small cathode to sample distances.

The model also shows that the dynamical evolution of the focusing distance allows to work with effective energy deposition pulses that are much shorter than those determined by the evolution of current and voltage.

\section{REFERENCES}

[1] G. W. McClure, "High voltage glow discharges in $\mathrm{D}_{2}$ gas-I. Diagnostics measurements," Phys. Rev., vol. 124, pp. 969-982, Nov. 1961.

[2] B. B. O'Brien, Jr., "Characteristics of a cold cathode plasma electron gun,” Appl. Phys. Lett., vol. 22, pp. 503-505, May 1973.

[3] H. F. Ranea-Sandoval, N. Reesor, B. T. Szapiro, C. Murray, and J. J. Rocca, "Study of Intense electron beams produced by high-voltage pulsed glow discharges," IEEE Trans. Plasma Sci., vol. PS-15, pp. 361-374, Aug. 1987.
[4] J. Christiansen and C. Schultheiss, "Production of high current particle beams by low pressure spark discharges," Z. Phys., vol A290, p. 35, 1979; also see, W. Benker, J. Christiansen, K. Frank, H. Gundel, and W. Hartmann et al., "Generation of intense pulsed electron beams by the pseudospark discharge," IEEE Trans. Plasma Sci., vol 17, pp. 754-757, 1989.

[5] R. A. Dugdale, "Soft vacuum processing of materials with electron beams," J. Mater. Sci., vol. 10, pp. 896-902, 1975.

[6] J. J. Rocca, J. D. Meyer, M. R. Farrell, and G. J. Collins, "Glow discharge electron beams: Cathode materials, electron gun design and technological applications," J. Appl. Phys., vol. 56, pp. 790-797, Aug. 1984.

[7] C. A. Moore, J. J. Rocca, G. J. Collins, P. E. Russell, and J. D. Geller, "Titanium disilicate formation by wide area electron beam irradiation," Appl. Phys. Lett., vol. 45, pp. 169-171, 1984.

[8] N. J. Ianno, J. T. Vedeyen, S. S. Chan, and B. G. Streetman, "Plasma annealing of ion implanted semiconductors," Appl. Phys. Lett., vol. 39, pp. 622-625, 1981.

[9] C. A. Moore, J. J. Rocca, T. Johnson, G. J. Collins, P. E. Russell, "Large area electron beam annealing," Appl. Phys. Lett., vol. 43, pp. 290-292, 1983.

[10] D. C. Bishop, K. A. Emery, J. J. Rocca, L. R. Thompson, H. Zarnani, G. J. Collins, "Silicon Nitride films deposited with an electron beam created plasma," Appl. Phys. Lett., vol. 44, pp. 598-600, 1984.

[11] L. R.Thompson, J. J. Rocca, P. K. Boyer, K. Emery, and G. J. Collins, "Electron beam assisted chemical vapor deposition of SiO," Appl. Phys. Lett., vol. 43, pp. 777-779, 1983.

[12] M. Hobel, J. Geek, G. Linker, and C. Schultheiss, "Deposition of superconductin YBACuO thin films by pseudospark ablation," Appl. Phys. Lett., vol 56, pp. 973-975, 1990.

[13] K. Kobashi, S. Miyauchi, K. Miyata, K. Nishimura, and J. J. Rocca, "Etching of polycrystalline diamond films by electron beam assisted plasma," J. Mater. Res., to be published.

[14] N. Mingolo and J. J. Rocca, "Production of amorphous metallic surfaces by means of a pulsed glow discharge electron beam," J. Mater. Res., vol. 7, no 5, pp. 1096-1099, 1992.

[15] S. A. Lee, U. A. Andersen, J. J. Rocca, M. Marconi, and N. D. Reesor, "Electric field distribution in the cathode sheath of an electron beam glow discharge," Appl. Phys. Lett., vol. 51, no. 6, pp. 409-411, 1987.

[16] J. I. Etcheverry and O. E. Martínez, "Monte Carlo calculation of the $\mathrm{keV}$ electron energy dissipation curves in compound materials," Ann. Argentine Chem. Soc., to be published.

[17] J. I. Etcheverry, "Numerical simulation of a laser melting and vaporization problem," Ann. Argentine Phys. Soc., vol. 5, pp. 220-223, 1993.

Javier Ignacio Etcheverry received the degree of Licenciado in mathematics and physics from the University of Buenos Aires, Buenos Aires, Argentina. $\mathrm{He}$ is currently working towards the Ph.D. degree in physics from the same university in the area of partial differential equations.

$\mathrm{He}$ is currently working in mathematical modeling and numerical simulation of interactions of energetic beams with materials.

Nélida Mingolo received the degree of Licenciado en Física (M.S.) and the Ph.D. degree in physics both from University of Buenos Aires, Buenos Aires, Argentina, in 1981, and 1992, respectively.

She has worked mainly on amorphous metals and fast-quenching techniques, as well as characterization techniques for metastable phases. She is presently Professor at the University of Buenos Aires and Head of the Directed Beam Laboratory, where she leads the development of the electron beam treatment of metallic surfaces.

Jorge J. Rocca (S'80-M'83-SM'94) photograph and biography not available at the time of publication.

Oscar Eduardo Martínez received the computador científico, the degree of Licenciado en Física (M.S.) and the Ph.D. in physics all from University of Buenos Aires, Buenos Aires, Argentina, in 1975, 1976, and 1982, respectively.

He has published more than 50 papers mainly on ultrashort pulse generation. $\mathrm{He}$ is currently a Full Professor at the University of Bueno Aires and a member of the Research Staff of Consejo Nacional de Investigaciones Científicas y Tecnológicas de la República Argentina.

Dr. Martínez is a fellow of the Optical Society of America. 